\title{
Perineural spread of pelvic malignancies to the lumbosacral plexus and beyond: clinical and imaging patterns
}

\author{
Stepan Capek, MD, ${ }^{1,2}$ Benjamin M. Howe, MD, ${ }^{3}$ Kimberly K. Amrami, MD, ${ }^{3}$ and \\ Robert J. Spinner, MD'1
}

Departments of ${ }^{1}$ Neurosurgery and ${ }^{3}$ Radiology, Mayo Clinic, Rochester, Minnesota; and ${ }^{22 n d ~ F a c u l t y ~ o f ~ M e d i c i n e, ~ C h a r l e s ~}$ University in Prague, Czech Republic

\begin{abstract}
OBJECT Perineural spread along pelvic autonomic nerves has emerged as a logical, anatomical explanation for selected cases of neoplastic lumbosacral plexopathy (LSP) in patients with prostate, bladder, rectal, and cervical cancer. The authors wondered whether common radiological and clinical patterns shared by various types of pelvic cancer exist.

METHODS The authors retrospectively reviewed their institutional series of 17 cases concluded as perineural tumor spread. All available history, physical examination, electrodiagnostic studies, biopsy data and imaging studies, evidence of other metastatic disease, and follow-up were recorded in detail. The series was divided into 2 groups: cases with neoplastic lumbosacral plexopathy confirmed by biopsy (Group A) and cases included based on imaging characteristics despite the lack of biopsy or negative biopsy results (Group B).

RESULTS Group A comprised 10 patients (mean age 69 years); 9 patients were symptomatic and 1 was asymptomatic. The L5-S1 spinal nerves and sciatic nerve were most frequently involved. Three patients had intradural extension. Seven patients were alive at last follow-up. Group B consisted of 7 patients (mean age 64 years); 4 patients were symptomatic, 2 were asymptomatic, and 1 had only imaging available. The L5-S1 spinal nerves and the sciatic nerve were most frequently involved. No patients had intradural extension. Four patients were alive at last follow-up.

CONCLUSIONS The authors provide a unifying theory to explain lumbosacral plexopathy in select cases of various pelvic neoplasms. The tumor cells can use splanchnic nerves as conduits and spread from the end organ to the lumbosacral plexus. Tumor can continue to spread along osseous and muscle nerve branches, resulting in muscle and bone "metastases." Radiological studies show a reproducible, although nonspecific pattern, and the same applies to clinical presentation.
\end{abstract}

http://thejns.org/doi/abs/10.3171/2015.7.FOCUS15209

KEY WORDS perineural spread; pelvic cancer; perineural invasion; lumbosacral plexopathy; imaging

I $\mathrm{T}$ is predicted that 453,360 new cases of pelvic malignancy (anorectal, genital, bladder, and ureteral cancer) will be diagnosed in the US in $2015 .{ }^{63}$ Lumbosacral plexopathy (LSP) occurs in $0.71 \%$ of all cancer patients; ${ }^{30}$ however, in the subgroup of pelvic cancer, it will be presumably higher.

Perineural spread of tumor from the organ to the lumbosacral plexus along the pelvic autonomic nerves has emerged as an alternate, logical explanation for select cases of neoplastic lumbosacral plexopathy (nLSP) in patients without extensive pelvic disease. This mechanism has been put forth for prostate, $, 9,17,27,37$ bladder, ${ }^{1}$ rectal, ${ }^{18}$ and cervical cancer ${ }^{28}$ We reviewed our institutional series of cases concluded as perineural spread of pelvic malignancy in search of clinical and radiological patterns.

\section{Methods}

After securing an approval from the institutional review board, we retrospectively reviewed our series of perineural spread of pelvic cancer. These were defined as cases of nLSP without extensive pelvic disease that demonstrated radiological signs of perineural tumor spread. These imaging signs were defined as enlarged lumbosacral plexus,

ABBREVIATIONS BNB = blood-nerve barrier; LSP = lumbosacral plexopathy; $\mathrm{nLSP}=$ neoplastic LSP; PNI = perineural invasion. 
spinal nerves or branching nerves (such as the sciatic nerves) on T1-weighted sequences that were hyperintense on T2-weighted sequences and demonstrated thick (perifascicular) enhancement on postgadolinium scans. Based on biopsy status we divided the patients into 2 groups: Group A, those in whom biopsy confirmed nLSP; and Group B, those in whom imaging characteristics demonstrated spread despite the lack of a biopsy being performed or negative biopsy results.

For both groups we recorded in detail all available demographic data (age and sex), history (original cancer, initial treatment, initial symptoms, duration, and character and laterality of symptoms), physical examination (pain, weakness, and sensory loss) and electrodiagnostic study findings, details of biopsy, findings on imaging studies including MRI, FDG PET/CT, and ${ }^{11} \mathrm{C}$-choline PET/CT, evidence of other metastatic disease, further oncological treatment, follow-up status, and appropriate time intervals.

Several cases from the series have been previously described as individual case reports. ${ }^{1,9,17,18,27,28,37}$ The purpose of this article is to review shared anatomical mechanisms and describe common radiological and clinical features.

\section{Results \\ Group A \\ History and Evaluation}

Ten patients ( 8 men and 2 women) were included in Group A (prostate cancer $n=5$, rectal cancer $n=2$, bladder cancer $n=2$, cervical cancer $n=1$ ). Clinical data for each patient are listed in Table 1 . The mean age was 69 years (range 48-85 years). Prior to LSP diagnosis, all patients except 1 underwent surgery, 4 patients received radiotherapy, 3 patients received hormonal therapy with leuprolide, and 2 patients received chemotherapy with other chemotherapeutic agents. Pain was the initial symptom in 7 patients, pain and weakness in 1 patient, and weakness in 1 patient; 1 patient was asymptomatic. In 1 patient the symptoms of LSP preceded the tumor diagnosis by 3 months; in the remaining symptomatic patients the mean time from the original tumor diagnosis to the initial symptom was 60.8 months (range 1 month-18 years). The asymptomatic patient had perineural spread that was discovered incidentally on follow-up imaging studies. On presentation, 9 symptomatic patients had a combination of pain, sensory loss, and weakness predominantly expressed in the L5-S1 dermatomes. Of 9 symptomatic patients, 8 had unilateral symptoms and 1 had bilateral symptoms. Electromyography (EMG) findings were abnormal in all patients available $(n=8)$ and demonstrated LSP in 6 patients and sciatic neuropathy in 2 . Six patients had no signs of distant metastatic disease at the time of the LSP diagnosis; 2 patients had metastases in lungs, 1 patient had metastases in the pelvis, and 1 had a solitary metastasis in the liver. All patients underwent biopsy procedures, which confirmed nLSP.

\section{Imaging}

Details of the imaging characteristics for all patients (n $=10$ ) are listed in Table 2. Occasionally, the nerves demonstrated a nodular appearance suggestive of localized tu- mor proliferation or perineural spread with "skip lesions." The sciatic nerve was involved in all 10 cases, and the obturator nerve showed signs of perineural spread in 1 patient. The most frequently affected spinal nerves were L-5 to $\mathrm{S}-2 ; 3$ patients had intradural extension of the tumor. MRI findings correlated with increased uptake on FDG PET/CT $(n=4)$ and ${ }^{11} \mathrm{C}$-choline PET/CT $(\mathrm{n}=3)$ scans. In 7 patients we found unilaterally thickened perirectal fascia, which was hyperintense on T2-weighted imaging and enhanced after intravenous contrast administration.

\section{Outcome}

Seven patients were alive at the time of the last followup. In 5 patients with no other metastases, further oncological treatment was initiated. In 3 the radiation therapy was escalated to include the lumbosacral plexus, 1 patient died before further therapy could be started, and in 1 patient information on follow-up therapy was not available. The mean time from the initial symptom to the last followup was 51 months (range 1 month-11 years).

\section{Group B}

History and Evaluation

Seven patients (6 men and 1 woman) were included in Group B (prostate cancer $n=4$; rectal cancer $n=2$, bladder cancer $\mathrm{n}=1)$. One patient only had images available; this patient is not included in the clinical data. Clinical data are given in Table 1 . The mean age was 64.2 years (range 32-81 years). Prior to LSP diagnosis all patients but 1 underwent surgery, 3 patients underwent radiotherapy, 2 patients received hormonal therapy with leuprolide, and 2 patients underwent chemotherapy with other chemotherapeutic agents. Pain was the initial symptom in 1 patient, pain and sensory loss in 1 patient, pain and weakness in 1 patient, and weakness in 1 patient; 2 patients were asymptomatic. The mean time from the original tumor diagnosis to the initial nerve symptom was 98 months (range 6 months-18 years). On presentation, 2 patients had a combination of pain, sensory loss, and weakness in the L5-S1 distribution, 1 patient had pain and sensory loss in the S-1 distribution, and 1 patient had only pain in the S-1 dermatome. All 4 symptomatic patients had right-sided symptoms; no bilateral disease was observed in Group B. EMG was performed in 2 patients and demonstrated LSP in both patients. One patient had distant metastases together with local spread of rectal cancer to the bladder, and $1 \mathrm{pa}-$ tient with prostate cancer had local spread to the rectum. Two patients underwent biopsy, the findings of which were negative for cancer. One patient deferred the biopsy.

\section{Imaging}

Details of the imaging characteristics for all patients $(n=7)$ are listed in Table 2 . The most frequently affected spinal nerves were L-5 to S-2; no patient had intradural extension of the tumor. MRI findings correlated with increased uptake on FDG PET/CT $(\mathrm{n}=3)$ and ${ }^{11} \mathrm{C}$-choline PET/CT $(n=1)$ scans. In 1 patient we found a unilaterally thickened perirectal fascia, which was hyperintense on T2-weighted imaging and enhancing after intravenous contrast; in 1 patient this finding was bilateral. 


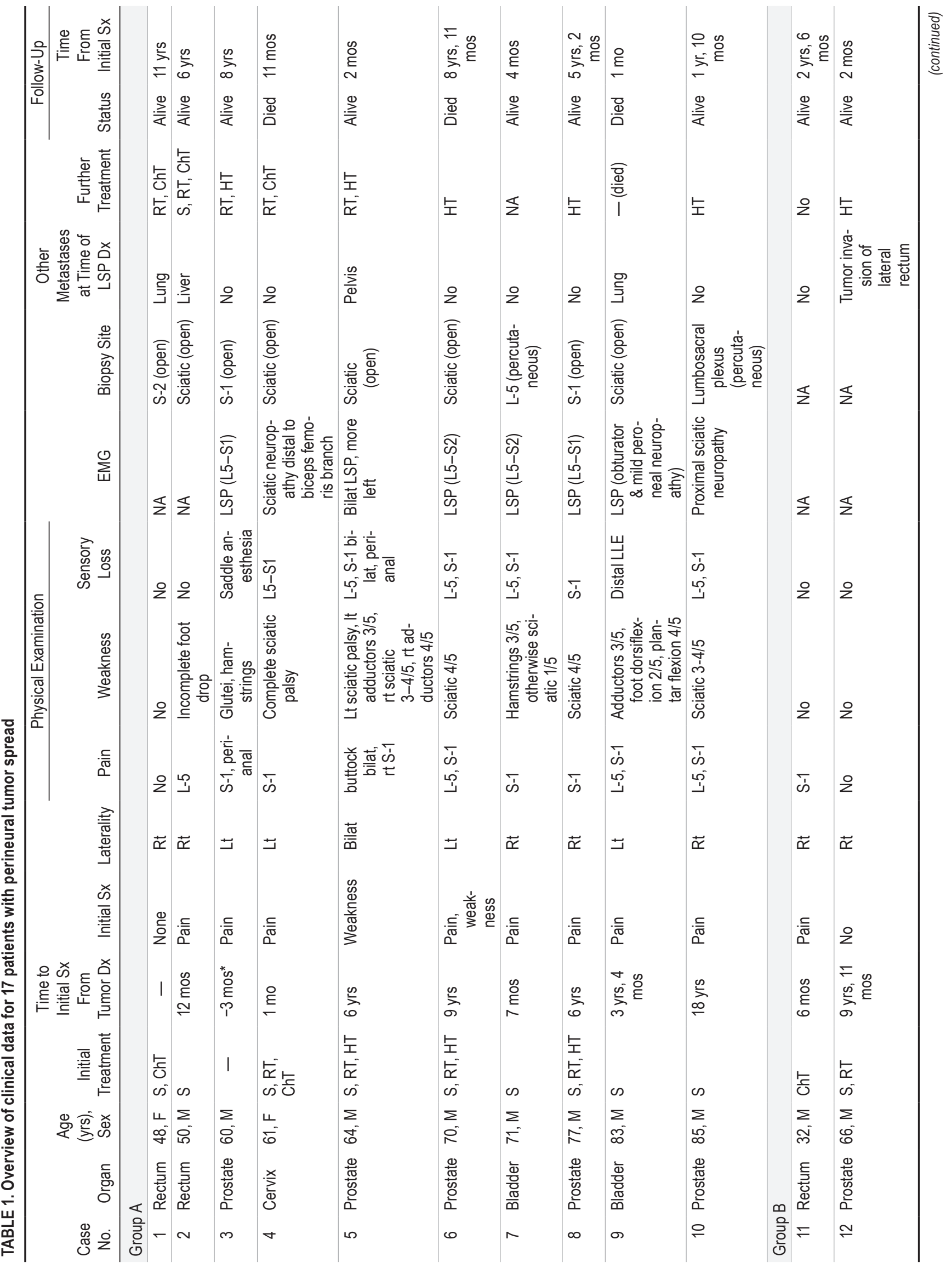




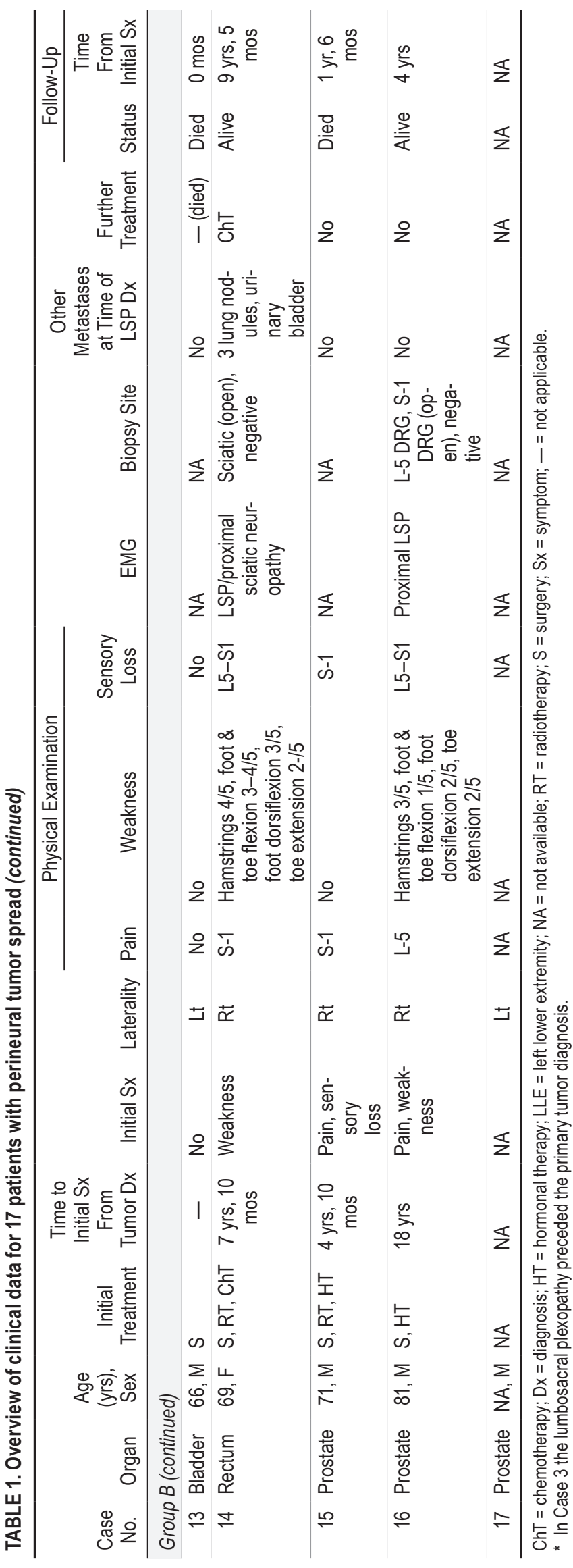

\section{Outcome}

Four patients were alive at the time of the last followup. In 3 patients with no other signs of recurrence or metastatic disease, no further therapy was administered. One of these patients later developed extensive pelvic disease and died a year later. Two patients continued hormonal or chemotherapy, and 1 patient died before further therapy could be started. The mean time from the initial symptom to the last follow-up was 41.8 months (range 2 months-9 years and 5 months).

\section{Discussion}

\section{Perineural Spread as a Cause of LSP}

Anatomy and Mechanism

In the pelvis, the autonomic visceral innervation is provided by the superior and inferior hypogastric plexuses. ${ }^{8,54,62}$ The superior hypogastric plexus is located below the aortic bifurcation and receives input from sympathetic lumbar splanchnic nerves. Caudally, it is connected to the inferior hypogastric plexus via paired hypogastric nerves. ${ }^{54}$ The inferior hypogastric plexus is a mixed sympathetic and parasympathetic plexus, which is innervated by the parasympathetic pelvic splanchnic nerves derived from the S2-4 spinal nerves and by the sympathetic sacral splanchnic nerves from the sacral sympathetic chain. The inferior hypogastric plexus extends to pelvic organs in the form of the rectal plexus, vesicoureteric plexus, and the uterovaginal plexus in females and prostate plexus in males. The pelvic portion of the ureters receives input from the sympathetic superior hypogastric plexus together with the trigone of the bladder. The ovarian plexus derived from T10-L1 innervates the ovaries and merges caudomedially with the uterovaginal plexus. ${ }^{62}$ The kidneys are supplied by the lateral prolongation of the celiac plexus called the renal plexus, which is derived from the T10-L1 levels. Boundaries between organ plexuses, especially those derived from the inferior hypogastric plexus, are more arbitrary rather than formed by obvious anatomical structures.

As demonstrated in prostate, ${ }^{37}$ bladder, ${ }^{1}$ rectal, ${ }^{18}$ and cervical ${ }^{28}$ cancer, the mechanism of spread is quite unified across different pelvic neoplasms (Fig. 1 and Video 1).

\footnotetext{
VIDEO 1. Animation of perineural spread. A short video demonstrates spread of prostate cancer (red), bladder cancer (blue), and rectal cancer (green) to the inferior hypogastric plexus and then the "common" pathway of spread (green) to the lumbosacral plexus, along the spinal nerves proximally (intradural extension is depicted). Perineural spread can also occur along the sciatic and superior gluteal nerves distally and then along the osseous branches to bony pelvis and along the nerve to the obturator internus to the muscle. Although the spread within and beyond the lumbosacral plexus is demonstrated in sequential steps, in vivo it occurs in all directions simultaneously. Used with permission of Mayo Foundation for Medical Education and Research. All rights reserved. Click here to view with Media Player. Click here to view with Quicktime.
}

The tumor cells infiltrate the inferior hypogastric plexus and spread to the lumbosacral plexus along the splanchnic nerves. From the lumbosacral plexus, cancer can spread distally to branching nerves or proximally to spinal nerves. This applies to the lateral walls and the dome of the bladder as well; tumors invading the bladder trigone spread pre- 
TABLE 2. Imaging data for 17 patients with perineural tumor spread

\begin{tabular}{|c|c|c|c|c|c|c|c|c|c|}
\hline \multirow[b]{2}{*}{$\begin{array}{c}\text { Case } \\
\text { No. }\end{array}$} & \multirow[b]{2}{*}{ Organ } & \multirow{2}{*}{$\begin{array}{l}\text { Age } \\
\text { (yrs), } \\
\text { Sex }\end{array}$} & \multirow[b]{2}{*}{ Laterality } & \multicolumn{4}{|c|}{ MRI } & \multirow[b]{2}{*}{ FDG PET/CT } & \multirow[b]{2}{*}{ Choline PET/CT } \\
\hline & & & & $\begin{array}{l}\text { Spinal } \\
\text { Nerves }\end{array}$ & $\begin{array}{c}\text { Peripheral } \\
\text { Nerves }\end{array}$ & $\begin{array}{l}\text { Intradural } \\
\text { Extension }\end{array}$ & $\begin{array}{l}\text { Route of } \\
\text { Spread* }^{*}\end{array}$ & & \\
\hline \multicolumn{10}{|c|}{ Group A } \\
\hline 1 & Rectum & $48, F$ & $\mathrm{Rt}$ & L5-S1 & Sciatic & No & Yes & NA & NA \\
\hline 2 & Rectum & $50, M$ & Rt & S-1 & Sciatic & No & No & NA & NA \\
\hline 3 & Prostate & $60, M$ & $\mathrm{Lt}$ & L5-S3 & Intrapelvic sciatic & No & Yes & NA & NA \\
\hline 4 & Cervix & $61, \mathrm{~F}$ & Lt & L5-S3 & Sciatic & No & Yes & S-2, sciatic & $\mathrm{NA}$ \\
\hline 5 & Prostate & $64, M$ & Bilat & $\begin{array}{l}\text { S2-3 bilat, } \\
\text { L4-S1 rt }\end{array}$ & Bilat sciatic & Yes & No & Bilat S-1 \& sciatic, It L-5 & Bilat S-1 \& sciatic, It L-5 \\
\hline 6 & Prostate & $70, M$ & $\mathrm{Lt}$ & L4-S4 & Sciatic & Yes & Yes & NA & NA \\
\hline 7 & Bladder & $71, \mathrm{M}$ & $\mathrm{Rt}$ & L5-S2 & Sciatic & No & Yes & NA & NA \\
\hline 8 & Prostate & $77, \mathrm{M}$ & $\mathrm{Rt}$ & S1-3 & Intrapelvic sciatic & Yes & No & NA & S-1 \\
\hline 9 & Bladder & $83, \mathrm{M}$ & $\mathrm{Lt}$ & L4-S1 & Obturator, sciatic & No & Yes & L4-S1 & NA \\
\hline 10 & Prostate & $85, \mathrm{M}$ & $\mathrm{Rt}$ & $\mathrm{S} 1-3$ & Sciatic & No & Yes & Sciatic & Sciatic \\
\hline \multicolumn{10}{|c|}{ Group B } \\
\hline 11 & Rectum & $32, \mathrm{M}$ & Rt & L5-S1 & Sciatic & No & Yes & NA & NA \\
\hline 12 & Prostate & $66, M$ & Rt & S2-4 & No & No & Yes & NA & Perirectal fascia \\
\hline 13 & Bladder & $66, M$ & $\mathrm{Lt}$ & L5-S1 & Sciatic & No & No & $\begin{array}{l}\text { Sciatic, bladder wall, pelvic } \\
\text { musculature, ham- } \\
\text { strings }\end{array}$ & $\mathrm{NA}$ \\
\hline 14 & Rectum & $69, \mathrm{~F}$ & Rt & L5-S1 & Sciatic & No & No & S-1, sciatic & NA \\
\hline 15 & Prostate & $71, \mathrm{M}$ & Rt & L5-S3 & Sciatic & No & No & S2-3, sciatic & Rt sciatic, rt S-2 \\
\hline 16 & Prostate & $81, M$ & Rt & L4-5 & Sciatic & No & No & NA & Negative \\
\hline 17 & Prostate & NA, M & $\mathrm{Lt}$ & L5-S2 & Sciatic & No & No & NA & $\mathrm{NA}$ \\
\hline
\end{tabular}

* Route of spread is represented on MRI studies as thickened perirectal fascia, which is hyperintense on T2-weighted images and enhancing after gadolinium contrast.

dominantly to the lumbar plexus along the lumbar splanchnic nerves and then can again extend beyond the plexus proximally to spinal nerves or even intradurally, or in the opposite direction to the obturator nerve. Theoretically, a similar mechanism could apply to ovarian, testicular, or renal cancer, although that has not been demonstrated yet. In 1 patient (Case 5) the tumor spread from the left spinal nerves to the contralateral spinal nerves, utilizing the dural sac as a bridge..$^{17}$

The underlying histological basis for perineural spread is perineural invasion (PNI). PNI is considered a major mode of prostate cancer extension outside the prostate gland $^{44}$ and is present in virtually all prostatectomy specimens with extracapsular spread. ${ }^{69} \mathrm{PNI}$ is present in up to $54.8 \%$ of surgically treated colorectal cancer cases,${ }^{75}$ up to $47.7 \%$ radical cystectomy specimens for bladder cancer, ${ }^{39}$ and up to $35.1 \%$ cervical cancer cases. ${ }^{20}$ White et al. ${ }^{74}$ suggested that these numbers might still be underreported unless special stains to highlight nerve tissue are used. The original definition proposed by Batsakis ${ }^{13}$ states that PNI is when the tumor is "in, around and through" the nerve. Liebig et al. ${ }^{41}$ proposed that finding tumor cells within any of the 3 layers of the nerve sheath represents PNI. Although the definition of PNI at the tumor site might be a subject for discussion, we consider definition of Liebig et al. sufficient for perineural spread. We advocate that perineural spread has occurred when tumor cells are found within any of the 3 layers of the nerve at a site distant from the tumor mass and acceptable anatomical explanation supported by indirect evidence (imaging) exists. We further advocate, although arguably, that perineural tumor spread also defines when tumor cells obviously track along the nerves, even without any nerve invasion.

Several theories to explain preferential invasion and growth along nerves have been proposed. The possibility that tumor cells simply follow the path of least resistance is unlikely. Various degrees of intraneural invasion are common, including cases solely confined to the endoneurium; ${ }^{28,37}$ the complex nerve ultrastructure of multiple layers of connective tissue and basal laminae makes intraneural invasion an unlikely path of least resistance. ${ }^{41}$ Two other mechanisms are more likely to explain the propensity of some tumors to PNI.

Endoneurium and Perineurium Are Chemically and Biologically Privileged Environments and Provide Sanctuary to Cancer Cells. The inner sanctum of the nerve is protected by the blood-nerve barrier (BNB) similarly as the brain is protected by the blood-brain barrier. ${ }^{49}$ The BNB is composed of several layers of perineurial cells encircling each fascicle and nonleaky capillaries surrounded by specialized pericytes. Perineurial cells are connected with tight junctions, and each cell is enveloped in a basement membrane. ${ }^{51}$ The formed perineurial barrier efficiently limits diffusion and cell penetration. Similarly, endoneurial capillaries are lined by nonfenestrated endothelium and are, as well as the pericytes, connected by tight junc- 


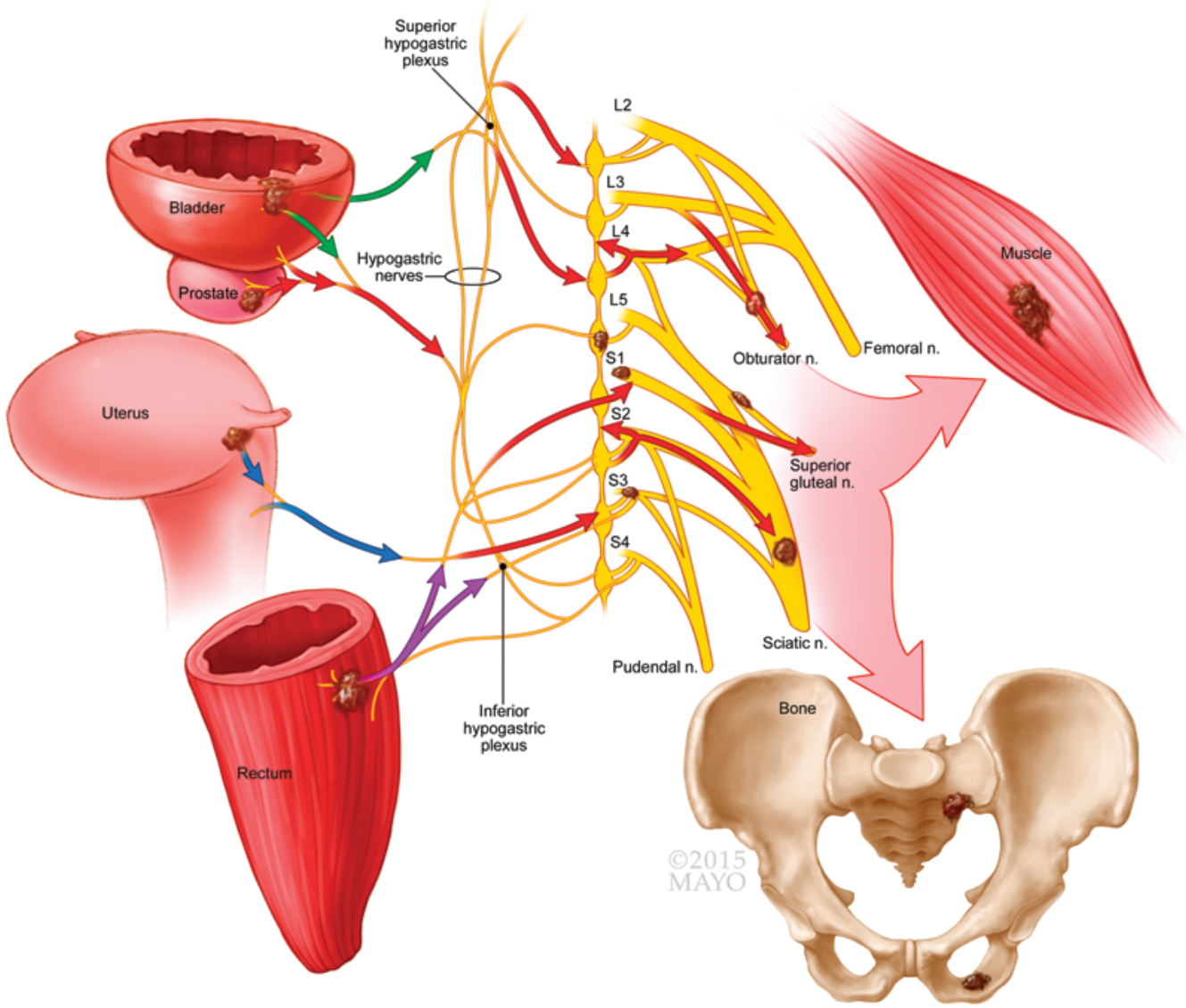

FIG. 1. Illustration demonstrating the unified mechanism of pelvic cancer spread from the prostate, rectum, bladder, and cervix to the lumbosacral plexus along the pelvic autonomic nerves (the superior and inferior hypogastric plexus) and then beyond the lumbosacral plexus to the bony pelvis and pelvic musculature along the osseous and muscle nerve branches. $n .=$ nerve. Used with permission of Mayo Foundation for Medical Education and Research. All rights reserved.

tions. ${ }^{58,61}$ The formed BNB creates an immunologically 34,46 and chemically protected environment. Not only this could prevent leukocyte infiltration into the tumor and chemotherapeutic agent delivery, but P-glycoprotein expressed by endothelial cells could be directly responsible for active drug efflux. ${ }^{3,56}$ The resulting "sheltering" is supported by cases of leukemia, which relapsed in peripheral nerves.

Direct Interactions Between Nerves and Cancer Cells Promote Tumor Growth and Survival. Recent research shows that more complex reciprocal signaling between nerves and tumor cells is involved. In prostate cancer, the nerves actively decrease tumor cell apopotosis ${ }^{4}$ and promote proliferation and migration through expressing $\mathrm{N}-\mathrm{Cam},{ }^{40}$ bystin, ${ }^{5}$ and Beta- $2^{32}$ adhesion molecules. The former could be mediated by antiapoptotic caveolin-I produced by the perineurium. ${ }^{6}$ This supports our and others ${ }^{311}$ observation that the perineurium is the most frequently involved layer (Fig. 2). On the other hand, prostate cancer cells ${ }^{7}$ and rectal cancer cells ${ }^{42}$ promote neurite outgrowth if murine dorsal root ganglion cells are co-cultured with the tumor cells. Most studies are focused on pancreatic ${ }^{11}$ and prostate cancer, and although we can presume that many cellular mechanisms are shared across various cancers, more research to elucidate PNI and perineural spread in pelvic malignancies is called for.

\section{Clinical Implications}

Neoplasms should always be included in the differential diagnosis as a possible cause of progressive LSP, especially in patients with a history of cancer. The primary pelvic malignancy diagnosis was preceded by nerve symptoms in only 1 patient (Case 3). In all other patients the time from the initial tumor diagnosis to LSP varied widely. All patients except for 1 in whom nLSP occurred after 1 year of follow-up after the tumor diagnosis underwent radiation therapy or chemotherapy (hormonal therapy) prior to the development of nLSP. We can presume that these interventions somewhat "salvaged" and delayed cancer progression within the nerves; however, our series is too small to perform any statistical evaluation of effect of different treatments. The risk of misdiagnosis is high, as multiple patients were referred to us as having radiation-induced plexopathy or inflammatory plexopathy. One patient underwent an L3-5 decompressive surgery and fusion at an outside hospital with no relief of symptoms.

In our series and other reported series ${ }^{31,67}$ of nLSP, the most common initial symptom was pain followed by weakness and sensory disturbances. On physical examination, findings from the straight-leg raising test can be positive. More indicative is percussion tenderness along the sciatic nerve if present. Infiltration of the pelvic sym- 


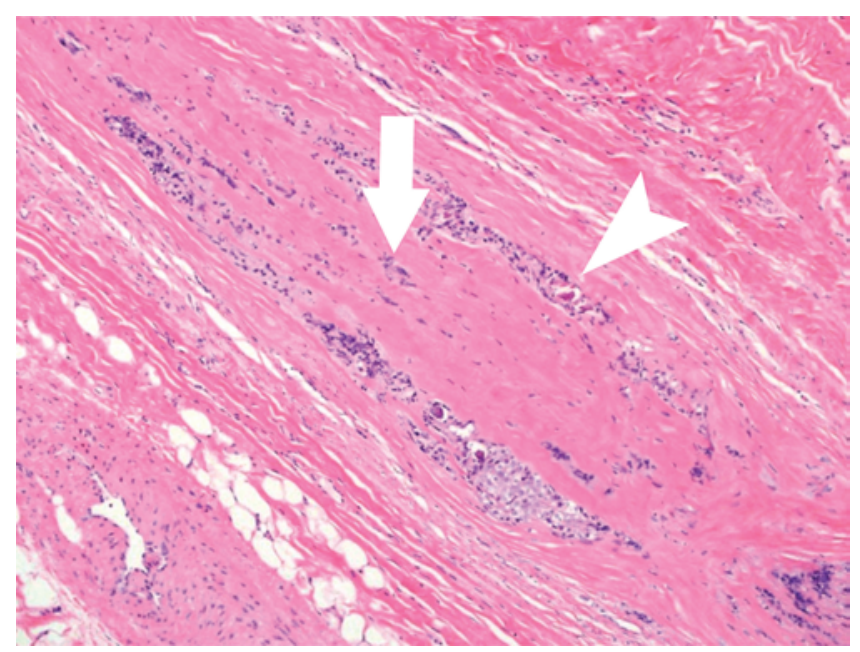

FIG. 2. Case 5. Perineural invasion (histopathology). Photomicrograph of a longitudinal section of a sciatic nerve fascicle demonstrating invasion of the endoneurium (arrow) and perineurium (arrowhead) with prostate cancer cells. The specimen was obtained from a fascicular biopsy. $H \& E$, original magnification $\times 100$.

pathetic system can produce "hot and dry foot" syndrome from the lack of perspiration and vasomotor activity. ${ }^{21,24}$ Electrodiagnostic studies can help to localize and objectify the extent of plexus involvement, and myokymic discharges, if present, strongly point toward radiation as the cause of LSP. ${ }^{67}$

\section{Imaging}

Imaging is the cornerstone of LSP evaluation. In perineural spread changes are often very subtle with no obvious tumor mass to help differentiation of neoplastic from nonneoplastic origin. Although numerous advanced MR sequences are advocated in the literature, an MRI diagnosis can often be made with standard imaging sequences performed with high resolution. This is best accomplished with 3-T imaging. Unilateral plexus imaging will increase imaging quality; however, we recommend a field of view that includes both plexuses from upper lumbar nerve roots to the sciatic nerve in the thigh. Otherwise, the extent of the disease can be underestimated for future therapy planning. Gradient recalled echo-based sequences (spoiled gradient recalled echo) and T2-based sequences (including fast spin echo) with robust fat suppression have become part of our standardized peripheral nerve imaging protocol in addition to standard T1-weighted sequences. The affected nerves are typically enlarged on T1-weighted sequences, often with irregular and nodular contours (Fig. $3 \mathrm{~A}-\mathrm{C})$. On T2-weighted images they are hyperintense (Fig. 3D-F), but the T2 signal might extend beyond tumor infiltration due to "downstream" effects. Fat-saturated postgadolinium contrast-enhanced sequences are highly valuable in the evaluation of perineural spread of disease (Fig. 3G-I) and should be performed unless there is a contraindication to gadolinium contrast administration. Affected nerves demonstrate thick, irregular, perifascicular enhancement. In 9 patients we observed an abnormality we consider to represent infiltrated inferior hypogastric plexus, i.e., spread from the organ to the sacral plexus. In 8 patients it was a thickened, hyperintense (on T2-weighted sequences) and heterogeneously enhancing perirectal fascia, unilaterally in 7 patients (Fig. 4A) and bilaterally in 1 patient (Case 12) (Fig. 4B). This simultaneous, "parallel" infiltration of both left and right inferior hypogastric plexuses provides an additional explanation for bilateral nLSP in addition to transdural spread described in Case 5 (Fig. 4C). ${ }^{17}$ In 1 patient we found a linear abnormality running more cranially toward the lumbar plexus. Consistent with the known innervation of the bladder, this patient presented with obturator nerve symptoms. ${ }^{1}$

Interpretation of the MRI after pelvic radiation may be challenging. In radiation-induced LSP the nerves might be enlarged but typically less than that seen in perineural tumor spread, hyperintense on T2-weighted images, and enhancing after gadolinium administration. The enhancement in radiation fibrosis is classically linear and homogeneous as opposed to the nodular and infiltrative appearance typical of PNI. The changes correspond to the radiation port. The nerves can be surrounded by fibrotic tissue producing fine, diffuse reticular enhancement, again confined to the irradiated area.

Positron emission tomography can be a very useful imaging method and support neoplastic nature if positive. No definite standard uptake value has been established in peripheral nerve evaluation; we use surrounding muscles as a reference. Our experience shows that in prostate cancer patients, studies using FDG provide superior sensitivity to ${ }^{11} \mathrm{C}$-choline scans (Fig. 5). This could be explained by the $\mathrm{BNB}$, which limits choline diffusion ${ }^{22}$ into the nerves or nonselective uptake of FDG by nonneoplastic (e.g., inflammatory cells). Bartels et al. ${ }^{12}$ reported a case very similar to ours with very avid uptake of FDG in the lumbosacral plexus, but which was concluded as inflammatory due to low positivity on the Choline PET/CT scan. We wonder if this case could in fact be neoplastic in nature.

\section{Diagnosis and Outcome}

The ultimate confirmation of tumor invasion is provided by biopsy, which we recommend to perform in all patients in whom a positive result might alter subsequent therapy. Whether to use an open or percutaneous approach is not clear. Open biopsy can help to select pathologically appearing tissue and increase yield, but it will increase risk..$^{14}$ Open biopsy also provides a larger sample and allows for more detailed evaluation of the degree of intraneural invasion. Important in biopsy site selection is high-quality imaging and accurate interpretation. Importantly, it should be kept in mind that a negative biopsy result does not exclude cancer, as demonstrated in Cases 14 and 16. Negative biopsy results can be explained by the relative occult nature of the neoplastic infiltration at operation, skip lesions, by biopsying the wrong portion of the nerve, or by associated pathology as acknowledged in Limitations. In 2 patients with negative biopsy results, inflammation with increase in endoneurial (Case 14) or surrounding (Case 16) collagen was present, but these changes were very nonspecific and did not allow us to conclude an alternate diagnosis. Percutaneous biopsy can on occasion be done of a "masslike" expansion of nerve (Case 7).

Once the diagnosis of nLSP is established, the treat- 

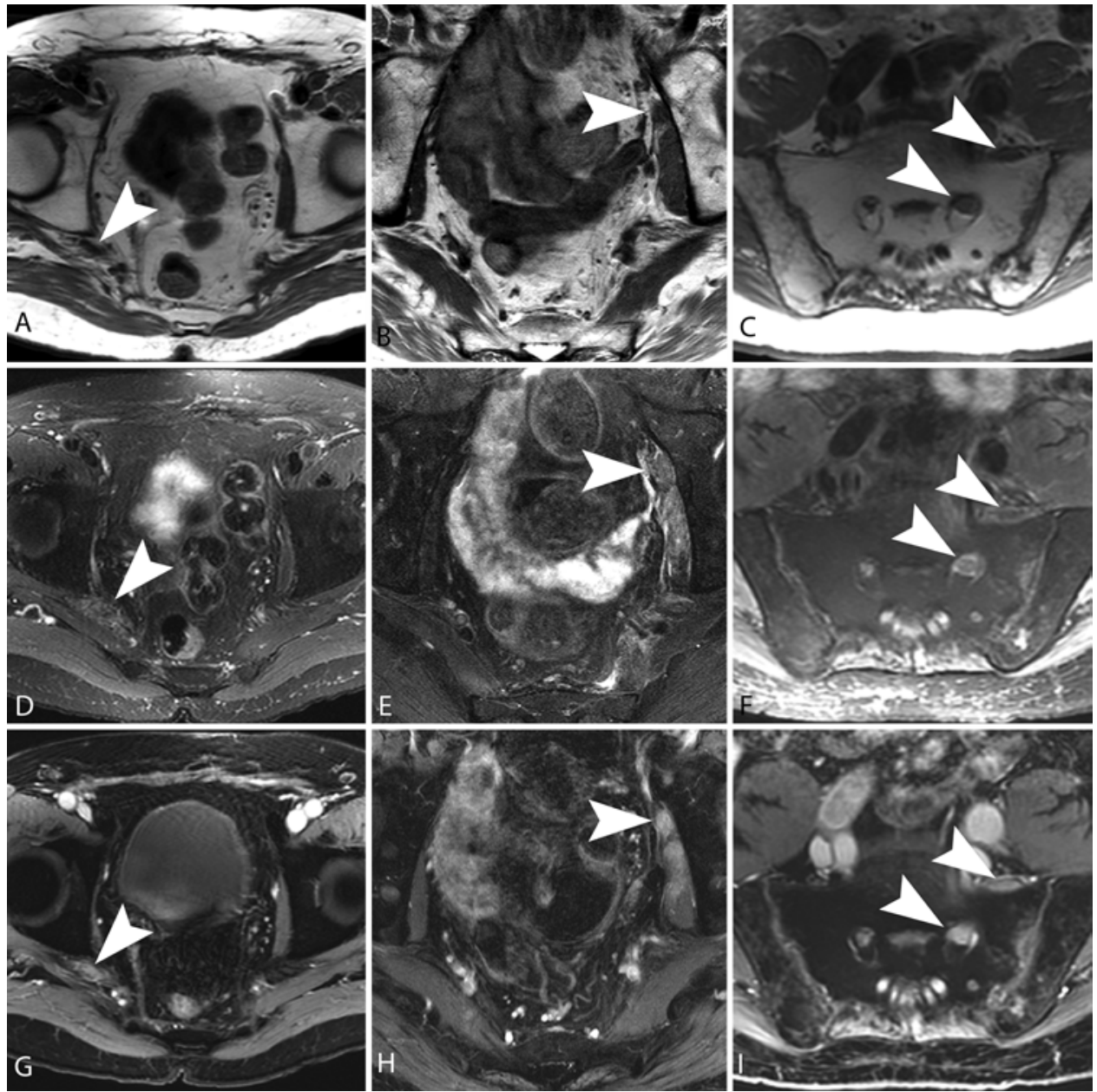

FIG. 3. MRI appearance of perineural tumor spread along the lumbosacral plexus. Axial T1-weighted images demonstrate enlarged sciatic (arrowhead in A), obturator (arrowhead in B), and L-5 and S-1 spinal nerves (arrowheads, C). Axial T2-weighted fat-suppressed images at the same levels show enlarged, hyperintense, and heterogeneous nerves (sciatic, obturator, spinal nerves, arrowheads in $\mathbf{D}, \mathbf{E}$, and $\mathbf{F}$, respectively). Axial spoiled gradient echo recalled images after intravenous gadolinium contrast visualize the same nerves at the same levels demonstrating avid, heterogeneous and preferential perifascicular enhancement (sciatic, obturator, and spinal nerves, arrowheads in $\mathbf{G}, \mathbf{H}$, and I, respectively).

ment currently does not change based on the underlying mechanism. Although visceral nerves could be sacrificed to some extent without a major impact on quality of life, the same cannot be said about major components of the LSP. In the biopsy-proven group (Group A), the diagnosis of nLSP led to therapy modification in 8 patients. In Group $\mathrm{B}$, no significant modification of therapy was made, but one patient who elected not to undergo biopsy later developed posterior pelvic wall recurrence and hydronephrosis and died a year later (Case 15). The role of the BNB in chemotherapy is yet to be established.

A review of the literature to explain reported prostate, rectal, bladder, and cervical cancers causing nLSP have been previously published. . $^{1,917,18,27,37}$ We wonder if our theory could explain nerve symptoms in other types of cancer that were not previously discussed. Uchida et al.$^{68}$ reported on a patient of uterine carcinoma who presented 4 years later with metastases to S-1 and S-2 spinal nerves and nerve roots. Cho et al. ${ }^{19}$ described a patient with a history of locally invasive squamous cell carcinoma of the anus. Ten months after diagnosis and resection, the patient pre- sented with weaker a quadriceps femoris muscle, diminished patellar and Achilles tendon reflexes, and intradural cauda equina metastasis. Johnson and Pomeroy ${ }^{33}$ reported 2 cases of Ewing's sarcoma that recurred at the margin of the radiation field "with tumor extending along the nerve roots of the lumbosacral plexus." Wald and Roland ${ }^{71}$ published a similar case of Ewing's sarcoma metastasis to a single nerve root, probably sensory, as stimulation did not produce any muscle contraction. Although kidneys are not exactly pelvic organs, several authors published cases suggestive of the same mechanism. Kubota et al. ${ }^{36}$ reported a case of renal cell carcinoma that metastasized to a single nerve root at the L-3 level; we wonder if this and several other cases of "metastatic" renal cell carcinoma to cauda equina ${ }^{2,25,43,52,65}$ could be explained by perineural spread.

\section{Perineural Spread Beyond the Lumbosacral Plexus}

Once tumor is within the lumbosacral plexus, it can continue to spread in the distal as well as proximal di- 


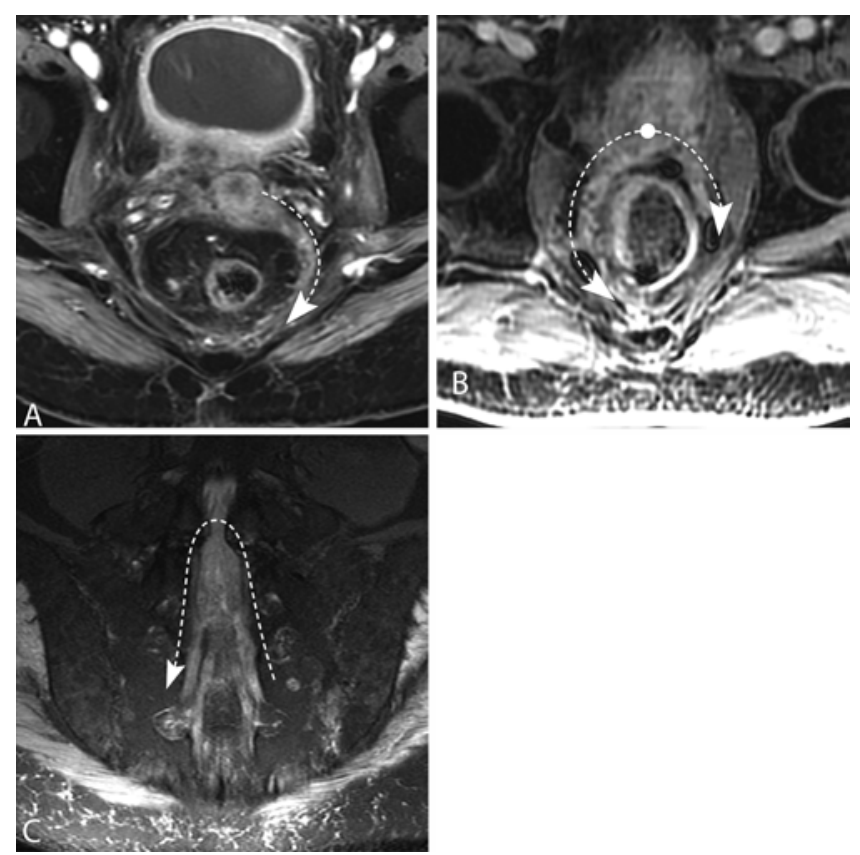

FIG. 4. Axial gadolinium-enhanced SPGR image of the pelvis, showing the unilaterally enlarged and enhancing perirectal fascia, presumably representing perineural spread of cervical cancer from the uterus to the lumbosacral plexus along pelvic autonomic nerves (dashed arrow in $\mathbf{A}$ ). Similar mechanism of spread can occur bilaterally (dashed arrows in B) and provide a theoretical explanation for bilateral symptoms in nLSP as demonstrated in an axial gadolinium-enhanced SPGR image of a patient with prostate cancer (B, the white dot represents the prostate gland). $A$ coronal T2-weighted fat saturated image demonstrates another mechanism of bilateral neoplastic plexopathy caused by perineural spread: a transdural spread of tumor between the opposing spinal nerves (dashed arrow in C).

rection. Perineural spread along the muscle and osseous nerve branches has been proposed as an alternate explanation for muscle $\mathrm{e}^{15}$ and bone ${ }^{16}$ metastases. This mechanism could explain cases of prostate cancer with nerve symptoms and regional bone metastases, ${ }^{26,50,70}$ or bladder with muscle mestastases. ${ }^{47}$ Although muscle is by nature very resistant to metastasis ${ }^{60}$ in these patients the resistance may decrease as the muscle becomes denervated.${ }^{73}$ Cancer cells can spread proximally onto the dural $\mathrm{sac}^{17}$ or even intradurally, which was observed in 3 patients in our series. Prostate-specific antigen levels in the CSF have been proposed to confirm and monitor CNS involvement in prostate cancer. ${ }^{48,59}$ We measured the prostate-specific antigen in CSF in Case 5, which was nearly 10 times higher than in serum. ${ }^{17}$ We theorize that cancer cells can subsequently seed within CSF, providing an alternate explanation for CNS metastases. Lefresne et al. ${ }^{38}$ reported a case of dura-based metastases at the T-4 and L4-5 levels with foraminal extension. Although the L4-5 metastasis was discovered later, we wonder if this case could be explained by perineural spread and subsequent intradural seeding, only with the L4-5 tumor lesion being somewhat salvaged and delayed by previous radiotherapy.

\section{Perineural Spread to Other Organs}

Warren et al. ${ }^{72}$ reported a case of prostate cancer with tumor masses lateral to the rectum with PNI and invasion of muscularis propria with PNI and even with tumor in anal submucosa, where PNI was "striking and fairly constant." Although the patient had locally advanced disease, it is clear that invasion of the rectum occurred preferentially along the nerves. We can report a similar observation in Case 12, although the diagnosis was only based on imaging (Fig. 6). If PNI is the main form of extraprostatic tumor extension, we wonder how many cases of bladder metastases of prostate cancer used nerves as a preferential mean of invasion, ${ }^{57}$ or how many cases of 1) cervical cancer metastases to the rectum or bladder, ${ }^{10} 2$ ) bladder cancer to the prostate, ${ }^{23,43}$ or 3) rectal cancer to the prostate or bladder ${ }^{53}$ occurred along the nerves.

\section{Perineural Spread as an Initial Step in Systemic Disease}

Kayahara et al. ${ }^{35}$ demonstrated perineural extension of pancreatic cancer to a lymph node, which could explain why lymph node metastases of pancreatic cancer correlate with PNI rather than with lymphatic vessel invasion..$^{66}$ Similar observations have been reported for prostate, ${ }^{64}$ rectal, ${ }^{29,55}$ and bladder ${ }^{39}$ cancer. We wonder if perineural extension could be an initial step to lymphogenous and potentially hematogenous spread thus leading to systemically disseminated disease.

\section{Limitations}

Although the perineural spread theory is the proposed anatomical explanation for cases in our series and selected cases in the literature, we acknowledge that other possible explanations exist. Cancer can spread hematogenously to very unusual locations including peripheral nerves. Our explanation applies to selected cases in which we find the imaging evidence very compelling. Although the endoneurium and perineurium lack lymphatic channels, ${ }^{41}$ we cannot exclude the possibility that the lymphatic system "approximated" cancer to the lumbosacral plexus. Another limitation is that 7 cases did not have histological confirmation, in which LSP might have been caused by unrelated pathology despite cancer history. We understand that final confirmation of our theory would be provided only by histopathological examination of the entire pathway from the organ to the plexus and beyond. Also, since we do not have the original radiation maps and we do not know what exact area was irradiated, we cannot comment on the efficacy of radiation therapy or propose any modifications in the initial oncological treatment.

\section{Conclusions}

We provide a unifying theory to explain LSP in selected cases of various pelvic neoplasms (prostate, bladder, cervical, and rectal cancer). Tumor cells can use splanchnic nerves as conduits and spread from the end organ to the lumbosacral plexus. From there, the cancer cells can continue proximally to the spinal nerves, intradurally, and to the contralateral plexus. Theoretically once they reach intradural space, they can seed and cause CNS metastases. Distally, the tumor spreads to the branching nerves as the sciatic or obturator nerves and along muscle and osseous branches to innervated muscles and bones. Clinically 

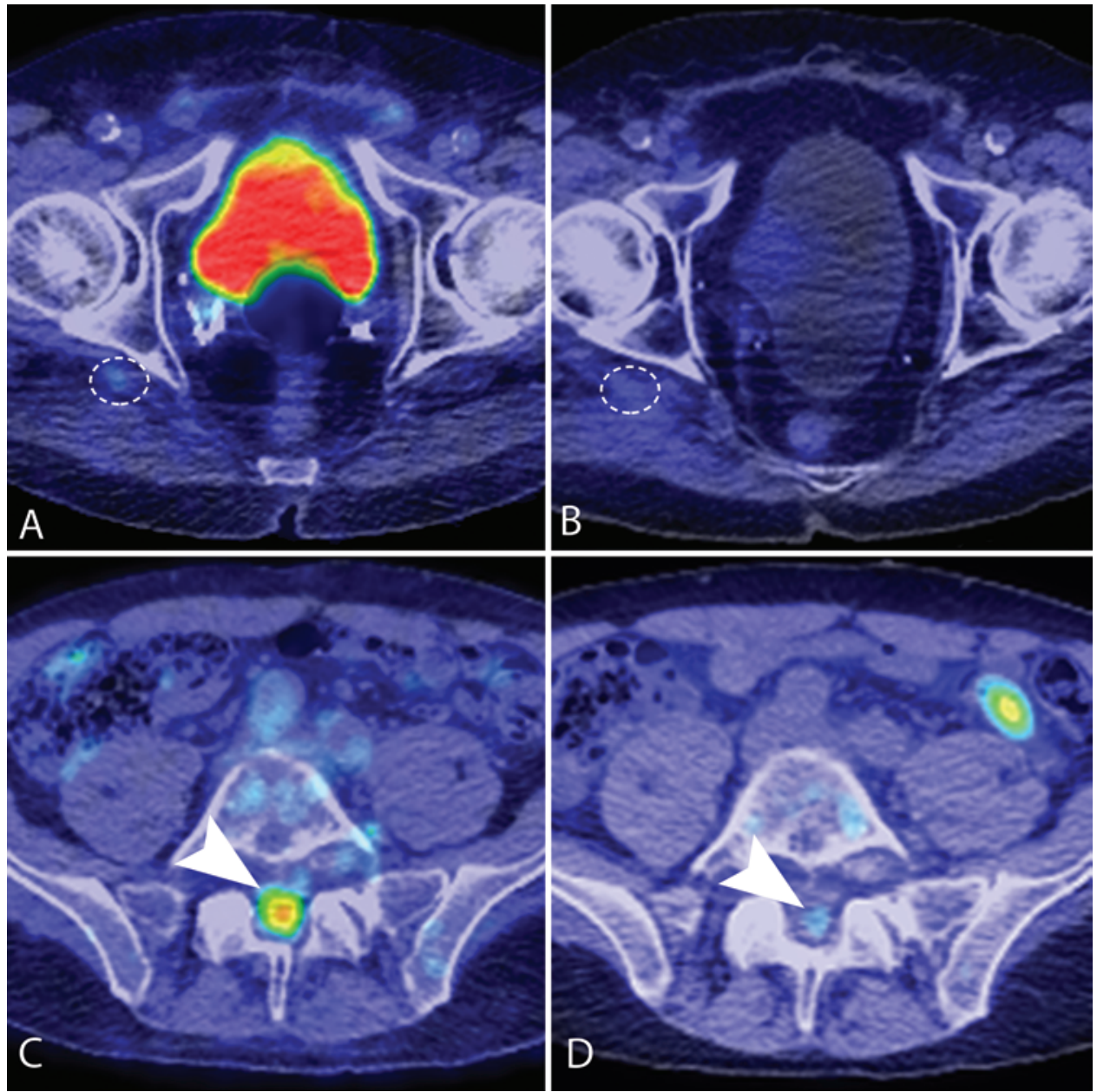

FIG. 5. FDG versus Choline PET/CT in perineural spread of prostate cancer. Based on our experience, in prostate cancer imaging FDG provides superior sensitivity to ${ }^{11} \mathrm{C}$-choline as demonstrated by axial composite FDG PET/CT (A and $\mathrm{C}$ ) and Choline PET/CT ( $B$ and D) images. The FDG PET/CT showed higher standard uptake values in the affected sciatic nerve (dashed circle in A) and in the intradural tumor extension (arrowhead in C) than comparable scans utilizing choline (dashed circle in B; arrowhead in D).

these patients present with pain followed by weakness and numbness, and although the clinical pattern is consistent with previously reported series of nLSP, in our series without significant tumor bulk the disease progressed sig-

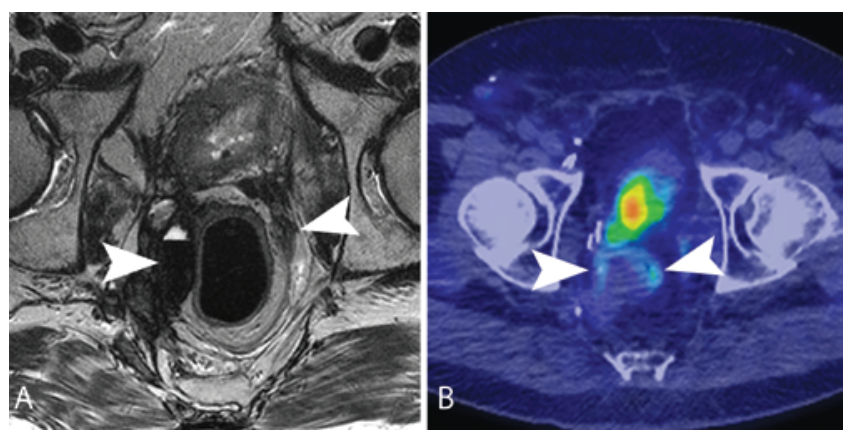

FIG. 6. Perineural spread of prostate cancer to the rectum. An axial gadolinium-enhanced LAVA-flex image demonstrates pararectal "metastases" of prostate cancer with invasion of rectal wall (arrowheads in A). An axial composite Choline PET/CT image shows increased uptake in the corresponding areas (arrowheads in $\mathrm{B}$ ). nificantly more slowly. Radiological studies show a subtle, reproducible pattern. We demonstrated that our proposed mechanism is applicable to other pelvic neoplasms (anal cancer, Ewing sarcoma) for which perineural spread has not been previously described.

\section{References}

1. Aghion DM, Capek S, Howe BM, Hepel JT, Sambandam S, Oyelese AA, et al: Perineural tumor spread of bladder cancer causing lumbosacral plexopathy: an anatomic explanation. Acta Neurochir (Wien) 156:2331-2336, 2014 [Erratum in Acta Neurochir (Wien) 157:153, 2015]

2. Alfieri A, Mazzoleni G, Schwarz A, Campello M, Broger M, Vitale M, et al: Renal cell carcinoma and intradural spinal metastasis with cauda equina infiltration: case report-part II. Spine (Phila Pa 1976) 30:260-262, 2005

3. Allt G, Lawrenson JG: The blood-nerve barrier: enzymes, transporters and receptors--a comparison with the bloodbrain barrier. Brain Res Bull 52:1-12, 2000

4. Ayala GE, Dai H, Ittmann M, Li R, Powell M, Frolov A, et al: Growth and survival mechanisms associated with perineural invasion in prostate cancer. Cancer Res 64:6082-6090, 2004 
5. Ayala GE, Dai H, Li R, Ittmann M, Thompson TC, Rowley $\mathrm{D}$, et al: Bystin in perineural invasion of prostate cancer. Prostate 66:266-272, 2006

6. Ayala GE, Dai H, Tahir SA, Li R, Timme T, Ittmann M, et al: Stromal antiapoptotic paracrine loop in perineural invasion of prostatic carcinoma. Cancer Res 66:5159-5164, 2006

7. Ayala GE, Wheeler TM, Shine HD, Schmelz M, Frolov A, Chakraborty S, et al: In vitro dorsal root ganglia and human prostate cell line interaction: redefining perineural invasion in prostate cancer. Prostate 49:213-223, 2001

8. Baader B, Herrmann M: Topography of the pelvic autonomic nervous system and its potential impact on surgical intervention in the pelvis. Clin Anat 16:119-130, 2003

9. Babu MA, Spinner RJ, Dyck PJ, Amrami KK, Nathan MA, Kawashima A, et al: Recurrent prostatic adenocarcinoma with perineural spread to the lumbosacral plexus and sciatic nerve: comparing high resolution MRI with torso and endorectal coils and F-18 FDG and C-11 choline PET/CT. Abdom Imaging 38:1155-1160, 2013

10. Badib AO, Kurohara SS, Webster JH, Pickren JW: Metastasis to organs in carcinoma of the uterine cervix. Influence of treatment on incidence and distribution. Cancer 21:434-439, 1968

11. Bapat AA, Hostetter G, Von Hoff DD, Han H: Perineural invasion and associated pain in pancreatic cancer. Nat Rev Cancer 11:695-707, 2011

12. Bartels AL, Zeebregts CJ, Enting RH, Slart RH: Fluorodeoxyglucose and $\mathrm{C}$-choline positron emission tomography for distinction of metastatic plexopathy and neuritis: a case report. Cases J 2:9323, 2009

13. Batsakis JG: Nerves and neurotropic carcinomas. Ann Otol Rhinol Laryngol 94:426-427, 1985

14. Capek S, Amrami KK, Dyck PJB, Spinner RJ: Targeted fascicular biopsy of the sciatic nerve and its major branches: rationale and operative technique. Neurosurg Focus 39(3):E12, 2015

15. Capek S, Amrami KK, Howe BM, Spinner RJ: Perineural tumor spread to the muscle: An alternative for muscle metastasis? Clin Anat 28:560-562, 2015 (Letter)

16. Capek S, Howe BM, Froemming AT, Amrami KK, Spinner RJ: Perineural spread in pelvic malignancies can be an alternate explanation for pelvic bony metastases rather than hematogenous spread. A report of two cases. Skeletal Radiol 44:1365-1370, 2015

17. Capek S, Howe BM, Tracy JA, García JJ, Amrami KK, Spinner RJ: Prostate cancer with perineural spread and dural extension causing bilateral lumbosacral plexopathy: case report. J Neurosurg 122:778-783, 2015

18. Capek S, Sullivan PS, Howe BM, Smyrk TC, Amrami KK, Spinner RJ, et al: Recurrent rectal cancer causing lumbosacral plexopathy with perineural spread to the spinal nerves and the sciatic nerve: an anatomic explanation. Clin Anat 28:136-143, 2015

19. Cho DY, Wang YC, Chen JT: Intradural metastasis to the cauda equina from carcinoma of the anus. Spine (Phila Pa 1976) 20:2341-2344, 1995

20. Cui L, Shi Y, Zhang GN: Perineural invasion as a prognostic factor for cervical cancer: a systematic review and metaanalysis. Arch Gynecol Obstet 292:13-19, 2015

21. Dalmau J, Graus F, Marco M: 'Hot and dry foot' as initial manifestation of neoplastic lumbosacral plexopathy. Neurology 39:871-872, 1989

22. DeGrado TR, Coleman RE, Wang S, Baldwin SW, Orr $\mathrm{MD}$, Robertson CN, et al: Synthesis and evaluation of 18Flabeled choline as an oncologic tracer for positron emission tomography: initial findings in prostate cancer. Cancer Res 61:110-117, 2001

23. Donat SM, Genega EM, Herr HW, Reuter VE: Mechanisms of prostatic stromal invasion in patients with bladder cancer: clinical significance. J Urol 165:1117-1120, 2001
24. Evans RJ, Watson CP: The hot foot syndrome: Evans' sign and the old way. Pain Res Manag 17:31-34, 2012

25. Gaetani P, Di Ieva A, Colombo P, Tancioni F, Aimar E, Debernardi A, et al: Intradural spinal metastasis of renal clear cell carcinoma causing cauda equina syndrome. Acta Neurochir (Wien) 146:857-861, 2004

26. Hansen JM, Rasti Z, Smith T, Lassen LH: Sciatic neuropathy as first sign of metastasising prostate cancer. BMJ Case Rep 2010:bcr1220092529, 2010

27. Hébert-Blouin MN, Amrami KK, Myers RP, Hanna AS, Spinner RJ: Adenocarcinoma of the prostate involving the lumbosacral plexus: MRI evidence to support direct perineural spread. Acta Neurochir (Wien) 152:1567-1576, 2010

28. Howe BM, Amrami KK, Nathan MA, Garcia JJ, Spinner RJ: Perineural spread of cervical cancer to the sciatic nerve. Skeletal Radiol 42:1627-1631, 2013

29. Huh JW, Kim HR, Kim YJ: Lymphovascular or perineural invasion may predict lymph node metastasis in patients with T1 and T2 colorectal cancer. J Gastrointest Surg 14:10741080,2010

30. Jaeckle KA: Neurological manifestations of neoplastic and radiation-induced plexopathies. Semin Neurol 24:385-393, 2004

31. Jaeckle KA, Young DF, Foley KM: The natural history of lumbosacral plexopathy in cancer. Neurology 35:8-15, 1985

32. Jansson KH, Castillo DG, Morris JW, Boggs ME, Czymmek KJ, Adams EL, et al: Identification of beta-2 as a key cell adhesion molecule in PCa cell neurotropic behavior: a novel ex vivo and biophysical approach. PLoS One 9:e98408, 2014

33. Johnson RE, Pomeroy TC: Evaluation of therapeutic results in Ewing's sarcoma. Am J Roentgenol Radium Ther Nucl Med 123:583-587, 1975

34. Kanda T: Biology of the blood-nerve barrier and its alteration in immune mediated neuropathies. J Neurol Neurosurg Psychiatry 84:208-212, 2013

35. Kayahara M, Nakagawara $H$, Kitagawa $H$, Ohta T: The nature of neural invasion by pancreatic cancer. Pancreas 35:218223, 2007

36. Kubota K, Akasu T, Fujita S, Sugihara K, Moriya Y, Yamamoto S: Clinical and pathological prognostic indicators with colorectal mucinous carcinomas. Hepatogastroenterology 51:142-146, 2004

37. Ladha SS, Spinner RJ, Suarez GA, Amrami KK, Dyck PJ: Neoplastic lumbosacral radiculoplexopathy in prostate cancer by direct perineural spread: an unusual entity. Muscle Nerve 34:659-665, 2006

38. Lefresne S, Fairchild A, Bistritz A, Venner P, Yee D: A case of indirect cauda equina syndrome from metastatic prostate cancer. Can Urol Assoc J 3:E31-E35, 2009

39. Leissner J, Koeppen C, Wolf HK: Prognostic significance of vascular and perineural invasion in urothelial bladder cancer treated with radical cystectomy. J Urol 169:955-960, 2003

40. Li R, Wheeler T, Dai H, Ayala G: Neural cell adhesion molecule is upregulated in nerves with prostate cancer invasion. Hum Pathol 34:457-461, 2003

41. Liebig C, Ayala G, Wilks JA, Berger DH, Albo D: Perineural invasion in cancer: a review of the literature. Cancer 115:3379-3391, 2009

42. Liebl F, Demir IE, Rosenberg R, Boldis A, Yildiz E, Kujundzic K, et al: The severity of neural invasion is associated with shortened survival in colon cancer. Clin Cancer Res 19:50-61, 2013

43. Mahadevia PS, Koss LG, Tar IJ: Prostatic involvement in bladder cancer. Prostate mapping in 20 cystoprostatectomy specimens. Cancer 58:2096-2102, 1986

44. Maru N, Ohori M, Kattan MW, Scardino PT, Wheeler TM: Prognostic significance of the diameter of perineural invasion in radical prostatectomy specimens. Hum Pathol 32:828833, 2001 
45. Maxwell M, Borges LF, Zervas NT: Renal cell carcinoma: a rare source of cauda equina metastasis. Case report. J Neurosurg 90 (1 Suppl):129-132, 1999

46. Moalem G, Monsonego A, Shani Y, Cohen IR, Schwartz M: Differential T cell response in central and peripheral nerve injury: connection with immune privilege. FASEB J 13:1207-1217, 1999

47. Nagao E, Nishie A, Yoshimitsu K, Irie H, Shioyama Y, Naito $\mathrm{S}$, et al: Gluteal muscular and sciatic nerve metastases in advanced urinary bladder carcinoma: case report. Abdom Imaging 29:619-622, 2004

48. Orphanos G, Ioannidis G, Michael M, Kitrou G: Prostatespecific antigen in the cerebrospinal fluid: a marker of local disease. Med Oncol 26:143-146, 2009

49. Orte C, Lawrenson JG, Finn TM, Reid AR, Allt G: A comparison of blood-brain barrier and blood-nerve barrier endothelial cell markers. Anat Embryol (Berl) 199:509-517, 1999

50. Paulson EC: Neoplasms of the bony pelvis producing the sciatica syndrome. Minn Med 34:1069-1074, 1951

51. Peltonen S, Alanne M, Peltonen J: Barriers of the peripheral nerve. Tissue Barriers 1:e24956, 2013

52. Petrelli F, Cabiddu M, Carpo M, Ghilardi M, Barni S: Progression of intramedullary metastasis during perioperative cessation of sunitinib. Nat Rev Urol 7:634-637, 2010

53. Rich T, Gunderson LL, Lew R, Galdibini JJ, Cohen AM, Donaldson G: Patterns of recurrence of rectal cancer after potentially curative surgery. Cancer 52:1317-1329, 1983

54. Rob L, Halaska M, Robova H: Nerve-sparing and individually tailored surgery for cervical cancer. Lancet Oncol 11:292-301, 2010

55. Saclarides TJ, Bhattacharyya AK, Britton-Kuzel C, Szeluga D, Economou SG: Predicting lymph node metastases in rectal cancer. Dis Colon Rectum 37:52-57, 1994

56. Saito T, Zhang ZJ, Shibamori Y, Ohtsubo T, Noda I, Yamamoto T, et al: P-glycoprotein expression in capillary endothelial cells of the 7th and 8th nerves of guinea pig in relation to blood-nerve barrier sites. Neurosci Lett 232:41-44, 1997

57. Saitoh H, Hida M, Shimbo T, Nakamura K, Yamagata J, Satoh T: Metastatic patterns of prostatic cancer. Correlation between sites and number of organs involved. Cancer 54:3078-3084, 1984

58. Sano Y, Shimizu F, Nakayama H, Abe M, Maeda T, Ohtsuki $\mathrm{S}$, et al: Endothelial cells constituting blood-nerve barrier have highly specialized characteristics as barrier-forming cells. Cell Struct Funct 32:139-147, 2007

59. Schaller B, Merlo A, Kirsch E, Lehmann K, Huber PR, Lyrer $\mathrm{P}$, et al: Prostate-specific antigen in the cerebrospinal fluid leads to diagnosis of solitary cauda equina metastasis: a unique case report and review of the literature. Br J Cancer 77:2386-2389, 1998

60. Seely S: Possible reasons for the high resistance of muscle to cancer. Med Hypotheses 6:133-137, 1980

61. Shimizu F, Sano Y, Maeda T, Abe MA, Nakayama H, Takahashi R, et al: Peripheral nerve pericytes originating from the blood-nerve barrier expresses tight junctional molecules and transporters as barrier-forming cells. J Cell Physiol 217:388-399, 2008

62. Shoja MM, Sharma A, Mirzayan N, Groat C, Watanabe K, Loukas M, et al: Neuroanatomy of the female abdominopelvic region: A review with application to pelvic pain syndromes. Clin Anat 26:66-76, 2013

63. Siegel RL, Miller KD, Jemal A: Cancer statistics, 2015. CA Cancer J Clin 65:5-29, 2015

64. Stone NN, Stock RG, Parikh D, Yeghiayan P, Unger P: Perineural invasion and seminal vesicle involvement predict pel- vic lymph node metastasis in men with localized carcinoma of the prostate. J Urol 160:1722-1726, 1998

65. Takada T, Doita M, Nishida K, Miura J, Yoshiya S, Kurosaka $\mathrm{M}$ : Unusual metastasis to the cauda equina from renal cell carcinoma. Spine (Phila Pa 1976) 28:E114-E117, 2003

66. Takahashi T, Ishikura H, Motohara T, Okushiba S, Dohke M, Katoh H: Perineural invasion by ductal adenocarcinoma of the pancreas. J Surg Oncol 65:164-170, 1997

67. Thomas JE, Cascino TL, Earle JD: Differential diagnosis between radiation and tumor plexopathy of the pelvis. Neurology $35: 1-7,1985$

68. Uchida K, Kobayashi S, Yayama T, Muramatsu J, Kurokawa T, Imamura Y, et al: Metastatic involvement of sacral nerve roots from uterine carcinoma: a case report. Spine J 8:849852,2008

69. Villers A, McNeal JE, Redwine EA, Freiha FS, Stamey TA: The role of perineural space invasion in the local spread of prostatic adenocarcinoma. J Urol 142:763-768, 1989

70. Vyshka G, Tartari F: S85 Osteosclerotic metastases of prostate cancer concomitant to peripheral motor neuropathy. Eur Urol Suppl 8:635-636, 2009

71. Wald SL, Roland TA: Intradural spinal metastasis in Ewing's sarcoma: case report and review of the literature. Neurosurgery 15:873-877, 1984

72. Warren S, Harris P, Graves R: Osseous metastasis of carcinoma of the prostate with special reference to the perineural lymphatics. Arch Pathol (Chic) 22:139-160, 1936

73. Weiss L: Biomechanical destruction of cancer cells in skeletal muscle: a rate-regulator for hematogenous metastasis. Clin Exp Metastasis 7:483-491, 1989

74. White M, Foulis AK, Smith G, Horgan PG, Roxburgh CS: The role of S100 staining in the pathological assessment of perineural invasion in rectal cancer. Colorectal Dis 16:7172,2014

75. Yang Y, Huang X, Sun J, Gao P, Song Y, Chen X, et al: Prognostic value of perineural invasion in colorectal cancer: a meta-analysis. J Gastrointest Surg 19:1113-1122, 2015

\section{Disclosure}

The authors report no conflict of interest concerning the materials or methods used in this study or the findings specified in this paper.

\section{Author Contributions}

Conception and design: all authors. Acquisition of data: all authors. Analysis and interpretation of data: all authors. Drafting the article: all authors. Critically revising the article: all authors. Reviewed submitted version of manuscript: all authors. Approved the final version of the manuscript on behalf of all authors: Spinner. Study supervision: all authors.

\section{Supplemental Information Videos}

Video 1, Media Player. http://mfile.akamai.com/21490/wmv/ digitalwbc.download.akamai.com/21492/wm.digitalsource-naregional/focus15-209_video_1.asx.

Video 1, Quicktime. http://mfile.akamai.com/21488/mov/ digitalwbc.download.akamai.com/21492/qt.digitalsource-global/ focus15-209_video_1.mov.

\section{Correspondence}

Robert J. Spinner, Mayo Clinic, 200 First St. SW, Gonda 8-214, Rochester, MN 55905. email: spinner.robert@mayo.edu. 